\title{
Global properties of magnetotail current sheet flapping: THEMIS perspectives
}

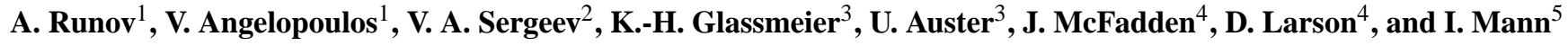 \\ ${ }^{1}$ Institute of Geophysics and Planetary Physics, UCLA, Los Angeles, CA-90095, USA \\ ${ }^{2}$ St. Petersburg State University, St. Petersburg, Russia \\ ${ }^{3}$ Institut für Geophysik und Extraterrestrische Physik, Technische Universität Braunschweig, Germany \\ ${ }^{4}$ Space Science Laboratory, University of California, Berkeley, CA, USA \\ ${ }^{5}$ University of Alberta, Edmonton, Canada
}

Received: 22 September 2008 - Revised: 10 December 2008 - Accepted: 10 December 2008 - Published: 19 January 2009

\begin{abstract}
A sequence of magnetic field oscillations with an amplitude of up to $30 \mathrm{nT}$ and a time scale of $30 \mathrm{~min}$ was detected by four of the five THEMIS spacecraft in the magnetotail plasma sheet. The probes $\mathrm{P} 1$ and $\mathrm{P} 2$ were at $X=-15.2$ and $-12.7 R_{E}$ and $\mathrm{P} 3$ and $\mathrm{P} 4$ were at $X=-7.9 R_{E}$. All four probes were at $-6.5>Y>-7.5 R_{E}$ (major conjunction). Multi-point timing analysis of the magnetic field variations shows that fronts of the oscillations propagated flankward (dawnward and Earthward) nearly perpendicular to the direction of the magnetic maximum variation $\left(B_{1}\right)$ at velocities of $20-30 \mathrm{~km} / \mathrm{s}$. These are typical characteristics of current sheet flapping motion. The observed anti-correlation between $\partial B_{1} / \partial t$ and the Z-component of the bulk velocity make it possible to estimate a flapping amplitude of 1 to $3 R_{E}$. The cross-tail scale wave-length was found to be about $5 R_{E}$. Thus the flapping waves are steep tail-aligned structures with a lengthwise scale of $>10 R_{E}$. The intermittent plasma motion with the cross-tail velocity component changing its sign, observed during flapping, indicates that the flapping waves were propagating through the ambient plasma. Simultaneous observations of the magnetic field variations by THEMIS ground-based magnetometers show that the flapping oscillations were observed during the growth phase of a substorm.
\end{abstract}

Keywords. Magnetospheric physics (Magnetotail; Plasma sheet; Plasma waves and instabilities)

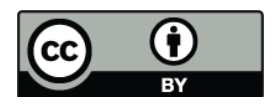

Correspondence to: A. Runov (arunov@igpp.ucla.edu)

\section{Introduction}

Flapping motion of the magnetotail current sheet manifests as variations of the magnetic field with an amplitude of up to several tens of $\mathrm{nT}$, often with a change of the magnetic field polarity, indicating the current sheet crossing. A temporal scale of these variations is rather wide, varying from $10 \mathrm{~s}$ to 10-20 min. Despite early spacecraft observations (Speiser and Ness, 1967), flapping motion still is not completely understood. Generally, the term "flapping" implies the up-down motion of the plasma/current sheet flux tubes with respect to unmoved spacecraft. Indeed, it was shown that $\partial B_{x} / \partial t$ anticorrelates with the north-south component $\left(V_{z}\right.$, the GSM coordinate system is used) of the plasma bulk velocity (Sergeev et al., 1998, 2003). This vertical motion may be induced by either variations of the interplanetary magnetic field (see Toichi and Miyazaki, 1976, and references therein) or by variations in the solar wind velocity and/or dynamic pressure (e.g. McComas et al., 1986). Recent global MHD simulations showed that even small-amplitude variations of the solar wind $V_{z}$ (only $6^{\circ}$ angular deviation of the solar wind from the $X Y$ plane) may produce a significant effect in the magnetotail, inducing global flapping with an amplitude of several $R_{E}$ (Sergeev et al., 2008). The up-down motion of the flux tubes may be also due to waves in the plasma sheet (Lui et al., 1978; Nakagawa and Nishida, 1989).

The association of flapping motion with the magnetospheric activity is long debated. Toichi and Miyazaki (1976) have found that flapping oscillations preferably occur in the early phases of substorms. This was confirmed by Sergeev et al. (1998), who showed that the majority of flapping events were observed within $10 \mathrm{~min}$ around substorm onsets or intensifications and during intervals of $K_{p} \geq+4$. Conversely,

Published by Copernicus Publications on behalf of the European Geosciences Union. 
a statistical survey of Geotail data showed that the majority of fast crossings of the current sheet occurred during low magnetic activity, however, at the $\mathrm{AE}$ increase phase (Sergeev et al., 2006b). A statistical analysis of the AL index during Cluster current sheet crossings also shows similar results: for 155 out of 266 crossings (i.e. more than $50 \%)$ the $\mathrm{AL}$ averaged over \pm 10 min-interval, centered on the crossing time, $(\langle A L\rangle)$ was larger than $-200 \mathrm{nT}$ for 73 crossings $-200>\langle A L\rangle>-500 \mathrm{nT}$, and only for 38 events out of $266\langle A L\rangle<-500 \mathrm{nT}$. Similar to the Geotail survey results, flapping crossings tend to occur near local $\mathrm{AL}$ minimum (maximum of AL absolute value). A survey of the $K_{p}$ values during the crossings shows that in $48 \%$ of events were during $K_{p}<2$, and in $30 \% 2<K_{p}<4$. Only in $22 \% K_{p}$ was found to be larger than 4 (compare with $3 / 4$ found by Sergeev et al., 1998). Thus, Cluster statistics do not show a clear dependence of flapping occurrence on the geomagnetic activity. Some statistical and eventual association of flapping with fast plasma flows in the plasma sheet was also shown (Sergeev et al., 2006b; Gabrielse et al., 2008). However, flapping oscillations without direct association with substorms and fast flows were also reported (Sergeev et al., 2003; Runov et al., 2005).

Multi-point observations allowing the wave front to be traced from one probe to the others are essential to understanding the mechanisms of flapping waves' generation and propagation. Cluster gave a possibility to determine the direction of the flapping front motion with respect to the spacecraft, to study electric current geometry during flapping, and to estimate a flapping waves amplitude. On a statistical basis as well as in the event analyzes, it was found that the magnetic field variations with an amplitude of $\delta B>15 \mathrm{nT}$ and the characteristic time scale of 30-300 s, referred to as flapping motion, are due to corrugation of the current sheet surface, i.e., surface waves, propagating flankward from the near-midnight sector of the magnetotail at a velocity of 30$100 \mathrm{~km} / \mathrm{s}$ (Zhang et al., 2002; Sergeev et al., 2004; Runov et al., 2005). The flankward propagation of the flapping corrugations indicates that these waves are most likely generated internally due to an intrinsic instability of the current sheet. However, global simulations showed that the dynamic response of the magnetotail current sheet to an increase the solar wind $V_{z}$ starts at the midnight meridian, but not near the flanks (Sergeev et al., 2008). Also, the internal generation of flapping oscillation in the plasma sheet might be externally triggered by a change in solar wind/IMF. Thus, the relationship between flapping and the solar wind and IMF dynamics remains an open question.

It is important to note that flapping waves, generally, are not periodic harmonic oscillations, but often exist as solitary folds on the sheet surface. The characteristic amplitude of these folds was found to be about $1 R_{E}$, and the characteristic length in the cross-tail direction is about $1-5 R_{E}$. Thus, the flapping fronts are rather steep and "nonlinear". The electric current in the flapping current sheet strongly deviates from the nominal plane geometry so that $j_{z}$ is often larger than $j_{y}$ and the current, locally, is almost vertical (Runov et al., 2005). It was also found that current sheet thickness substantially decreases on the flapping fronts (Runov et al., 2006). Analysis of the magnetic field configuration on the strongly tilted flapping fronts reveals that they are formed by a vertical slippage of a certain volume of the plasma sheet with respect to neighboring ones (Petrukovich et al., 2003, 2006). This complex geometry of the flapping current sheet is in agreement with results of several theoretical models of flapping waves (Golovchanskaya and Maltsev, 2004; Erkaev et al., 2008).

The aforementioned "slippage"-mode model suggests certain global properties of flapping: the flapping-related corrugations on the sheet surface are supposed to be tail-elongated structures with the tail-aligned scale much larger than the cross-tail one. A study of the global-scale (of $10 R_{E}$ ) properties of the flapping waves (such as the tail-elongation) requires a constellation with the tail-aligned separation of several to $10-15 R_{E}$. Event studies performed with the pair of Geotail and Interball, radially separated by $\sim 10 R_{E}$ (Petrukovich et al., 2003) and during the Cluster - Double Star TC-1 conjunction with a radial separation of $5 R_{E}$ (Zhang et al., 2005) indeed showed correlated magnetic field variations. However, studies with equatorial-orbiting fleet are still needed to prove the model.

The THEMIS mission employing five identical spacecraft (probes) carrying a comprehensive set of instruments as well as the dense network of ground-based observatories equipped by magnetometers and all-sky cameras (Angelopoulos, 2008), provides wide possibilities to study global properties of the magnetotail current sheet flapping waves and their counterparts in the ionosphere and groundmeasured magnetic field. A THEMIS tail-aligned conjunction with probe separation of several $R_{E}$ along $X$ and of $2 R_{E}$ in the cross-tail $(Y)$ direction is the ideal configuration to figure out whether the flapping fronts are indeed tail-aligned.

In this paper we report on the THEMIS major conjunction event study, providing an example of magnetotail current sheet flapping as seen by the tail-elongated fleet with the separation of $\sim 10 R_{E}$.

\section{Data analysis}

In the following sections, we discuss the magnetotail current sheet dynamics during 04:00-09:00 UT on 20 December 2007. Figure 1 shows an overview plot of the space and ground-based observations during the interval of interest. The solar wind velocity and dynamic pressure were obtained from WIND OMNI measurements at $[256.3,-0.2$, 24.2] $R_{E}$, recalculated to $1 \mathrm{AU}$. The $X$ (GSM coordinates) component of the solar wind bulk velocity fluctuated between -500 and $-600 \mathrm{~km} / \mathrm{s}$; the $Y$ component was generally positive (duskward), fluctuating between -10 and $40 \mathrm{~km} / \mathrm{s}$; 


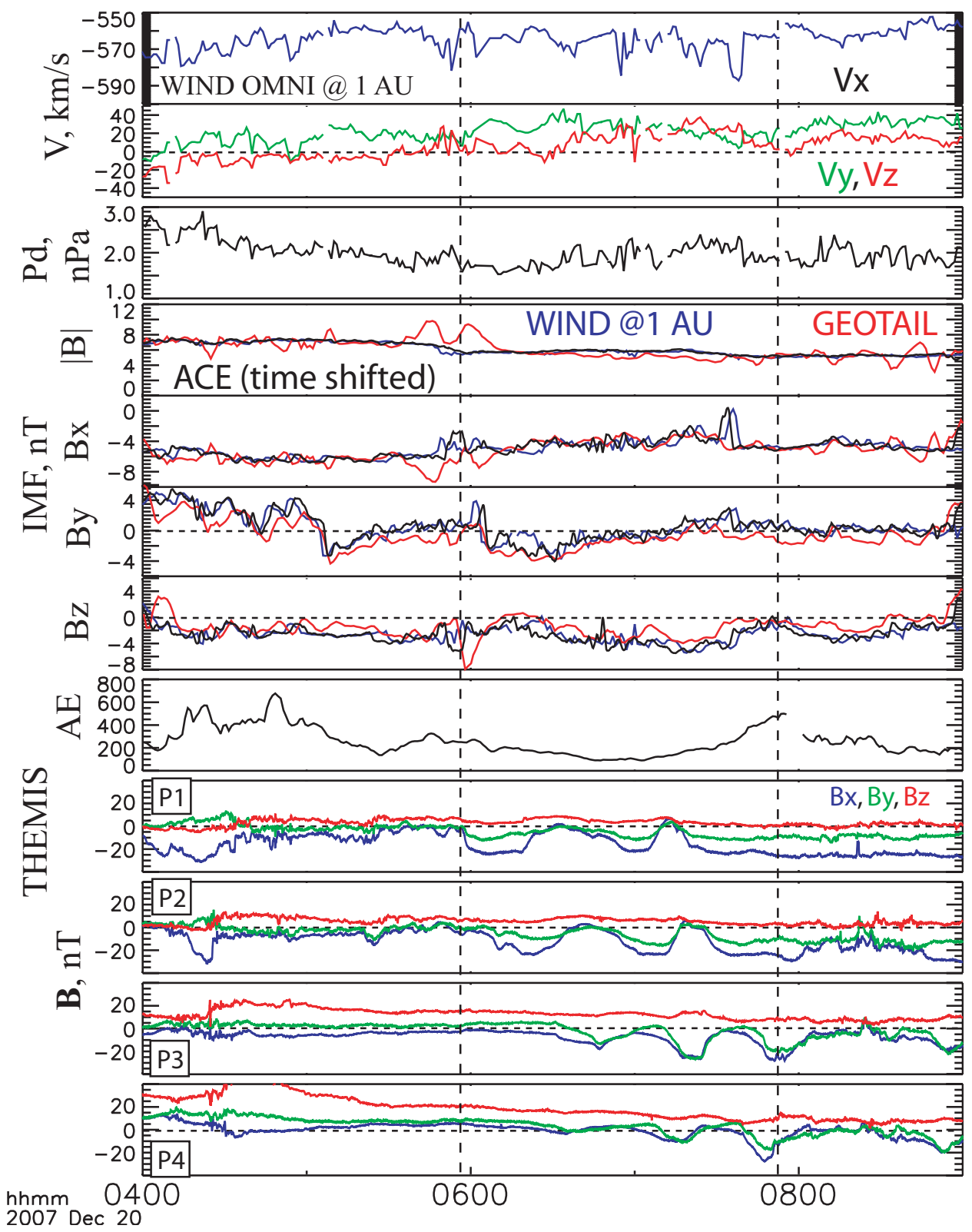

Fig. 1. Observations during 04:00-09:00 UT on December 20, 2007. From top to bottom: the X-component of solar wind bulk velocity, $Y$ and $Z$ GSM components of the solar wind velocity, and solar wind dynamic pressure from the WIND satellite, the IMF strength, and GSM components of the IMF from WIND; THEMIS pseudo-AE index; magnetic field (GSM) from the four THEMIS probes (P1, P2, P3 and P4) versus UT.

the Z-component reversed from southward to northward at about $05: 45 \mathrm{UT}$ and varied between -10 and $40 \mathrm{~km} / \mathrm{s}$. The solar wind dynamic pressure gradually decreased from 2.8 to $1.7 \mathrm{nPa}$ between 04:00 and 06:00 UT, then stayed fluctuating between 1.7 and $2.7 \mathrm{nPa}$. Measurements of the interplanetary magnetic field (IMF) by ACE at $[219.9,-26.6,18.2] R_{E}$ time shifted by $1900 \mathrm{~s}$, WIND (recalculated to $1 \mathrm{AU}$ ), and Geotail at $[8.04,-17.78,-1.18] R_{E}$ are presented in Fig. 1. Although general trends in IMF strength and components are similar at the three satellites, a pronounced difference is vis- ible between 05:45-06:05 UT. The IMF $B_{y}$ varied between -3 and $5 \mathrm{nT}$, and the IMF $B_{z}$ was predominantly southward (after a southward turning at 04:00-04:15 UT) until 09:00 UT, varying between -5 and $0 \mathrm{nT}$.

THEMIS pseudo-AE index, calculated using the THEMIS array of ground-based magnetometers (Mende et al., 2008; Mann et al., 2008), shows three major activations with maxima of 700, 300, and 600 nT at 04:30, 05:50 and 08:00 UT, respectively. The minimum of the THEMIS AE during the interval of interest was about $100 \mathrm{nT}$. 


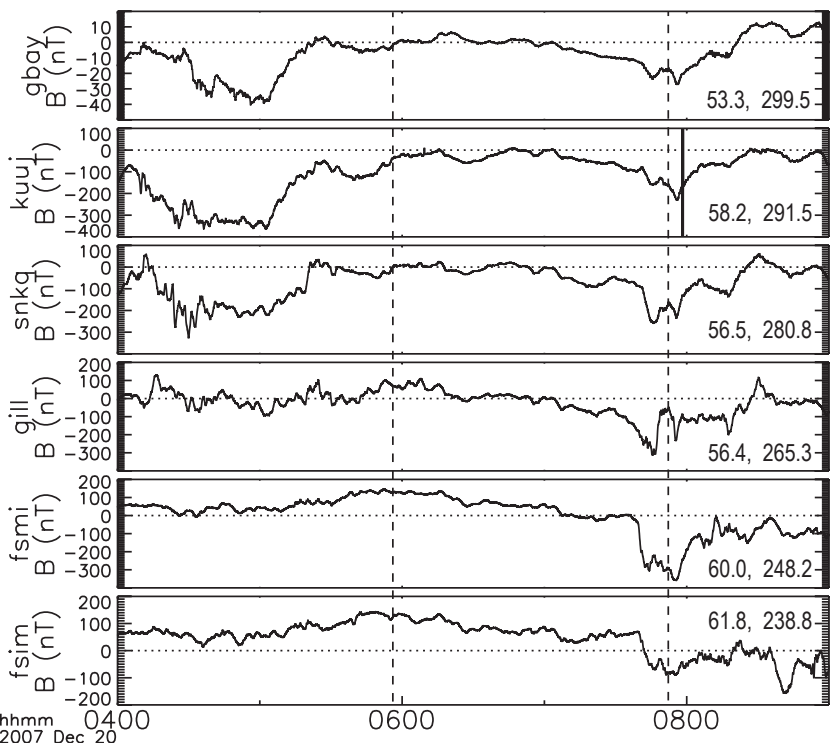

Fig. 2. Ground-based magnetic field observations during 04:0009:00 UT. From top to bottom: $B_{x}$ component (northward) with daily median value subtracted measured by THEMIS GBO from east to west along the THEMIS ground track on the Northern Hemisphere. Vertical dashed bars bound the interval of flapping, observed by THEMIS probes. Geographic coordinates of the stations are specified.

Four bottom panels show the magnetic field from THEMIS Flux Gate Magnetometer (FGM Auster et al., 2008) at four probes THB (P1), THC (P2), THD (P3) and THE (P4). The positions of THEMIS spacecraft in the $X Y$ and $X Z$ GSM planes at 07:00 UT are shown in Fig. 3 (top panel). $\mathrm{P} 1, \mathrm{P} 2$, and $\mathrm{P} 3$ probes were aligned along $X$ within $6.5<Y<7.5 R_{E}$ (major conjunction). At 07:00 UT, P1 and $\mathrm{P} 2$ were at $X=-15.2$ and $-12.7 R_{E}$, respectively. P3 and P4 were at the same $X=7.9 R_{E}$ separated in $Y$ by $1.3 R_{E}$ ( $Y=-7.5$ and $-6.2 R_{E}$, respectively). All four probes were in the Southern Hemisphere, within $5 R_{E}$ from the nominal equator $\left(Z=-4.9,-4.2,-2.9\right.$, and $-2.8 R_{E}$ at $\mathrm{P} 1, \mathrm{P} 2, \mathrm{P} 3$, and $\mathrm{P} 4$, respectively). A well pronounced train of the quasiperiodical magnetic field oscillations was observed by all four probes between 05:55 and 07:55 UT. Amplitudes of the $B_{x}$ variations at the two tailward probes (P1 and $\left.\mathrm{P} 2\right)$ achieved 25-30 nT. At the two near-Earth probes (P3 and P4), the amplitudes were smaller and with a tendency to grow in time. Oscillations in $B_{y}$ were in phase and with similar amplitude with those of $B_{x}$. Although the period of oscillations was about 20-40 min, current sheet crossings (when $B_{x}$ changes from 0 to $\approx-15 \mathrm{nT}$ ) were significantly shorter (especially at P1), showing a properties of a quasi-rectangular wave rather that a sinusoidal one.

Ground-based magnetometer observations from east to west along the THEMIS ground track on the Northern Hemisphere are presented in Fig. 2. The traces of the northward

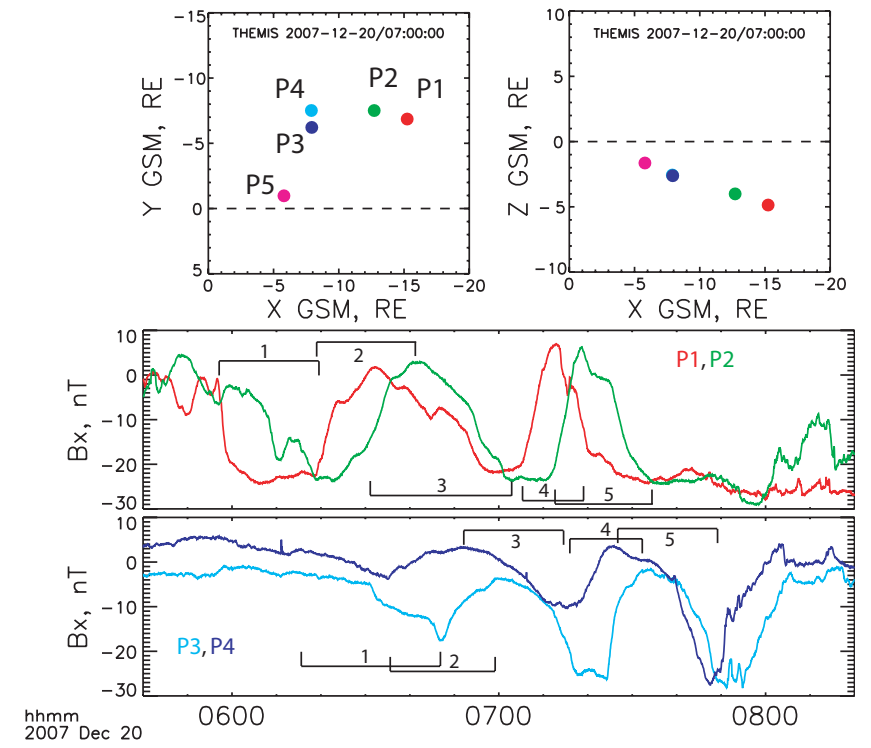

Fig. 3. Positions of THEMIS spacecraft $X Y$ and $X Z$, GSM planes at 07:00 UT on 20 December 2007 (top panel); The X-component of the spin-averaged magnetic field measured by P1 and P2 (mid panel) and P3 and P4 (bottom panel) between 05:40 and 08:20 UT on 20 December 2007.

magnetic field component $\left(B_{x}\right)$ at the stations in the premidnight sector (GILL, FSMI, FSIM) show a similar tendency to decrease during the flapping interval, marked by vertical dashed bars. This tendency is less obvious at the stations in the post-midnight sector (GBAY, KUUJ, SNKQ). No distinct variations in the $\mathrm{Pi} 2$ range were detected between 05:50 and 07:50 UT. $B_{x}$ traces at all stations show a set of negative variations with amplitudes of $\sim 50 \mathrm{nT}$ and a timescale of 10 to $30 \mathrm{~min}$.

Figure 3 shows time series of $B_{x}$ measured by two distant probes (P1 and $\mathrm{P} 2)$ and by two near-Earth probes (P3 and $\mathrm{P} 4)$ in two separate panels. The similarity in shapes of the time series is visible: the probes detected propagating spatial structures. In the distant pair, the $B_{x}$ undulations were detected first by $\mathrm{P} 1$ then by $\mathrm{P} 2$. Most notable is the structure \#4-5: it propagated from P1 to P2 within $\sim 10$ min (peakto-peak) without large changes in its shape. Since P1 was situating tailward and duskward from P2, the wave was propagated Earthward and dawnward. The distance between the two probes in the $X Y$ plane was $2.6 R_{E}$, thus, the half-width of the structure is about $2-3 R_{E}$. In the near-Earth pair, the fronts were detected first by P4 then by P3, indicating dawnward propagation of the disturbance (Fig. 3, top panel).

To obtain the quantitative information on the propagation direction of current sheet flapping fronts in the equatorial magnetotail, the Minimum (Maximum) Variance Analysis (MVA, Sonnerup and Scheible, 1998) and the multipoint timing analysis of the magnetic field time series were performed. Applying the multi-point timing analysis, we 
assume that all four probes detected the same planar wave fronts. The 2-component vector of the front velocity may result from 3-point timing (e.g. Gabrielse et al., 2008). In this case the solution of the resulting linear equation system is unique (e.g. Harvey, 1998). 4-point timing gives an overdetermined linear equation system. The singular value decomposition technique (Press et al., 1992) was used to solve it. Applied to the magnetic field traces at P1 (P2), P3, and P4 (3-point timing) and those at P1, P2, P3, and P4 (4-point timing) during the flapping oscillations, both methods gave results with an excellent agreement. The eigenvalues $\lambda_{2}$ and $\lambda_{3}$ resulting from MVA for almost all crossings were of the same order of value, and therefore, intermediate and minimum variance directions were not distinguishable. Thus, MVA was used mainly to determine the maximum variance direction. For a few crossings, however, $\lambda_{2} / \lambda_{3}>3$, allowing to resolve the minimum variance direction. The MVA results for these crossings are summarized in Table 1. The presented results show that the minimum variance direction (interpreted as the current sheet normal) often deviates from the nominal $\left(Z_{\mathrm{GSM}}\right)$ direction, indicating a tilt in the plane, perpendicular to the main magnetic field (maximum variance, $\mathbf{R}_{1}$ ) direction. This effect is more clear in mid-tail plasma sheet (at P1 and P2). In the near-Earth tail, during the first and the second crossing by P3, the normal was directed mainly along $Z_{\mathrm{GSM}}$. However, for the larger-amplitude flapping (\#3, 4, and 5, see Fig. 3) MVA of P3 and P4 time series shows the large tilt in the $\mathbf{R}_{1}$-perpendicular plane. The large tilt of the normal to the cross-tail plane was reported to be a distinctive feature of flapping motion (e.g. Sergeev et al., 2006b). It is important to note that for large-tilt cases the "guide"-component of the magnetic field $\left(B_{2}\right)$ is typically larger than the normal one $\left(B_{3}\right)$, which is consistent with the "slippage"-mode flapping (Petrukovich et al., 2006; Sharma et al., 2008).

Figure 4 shows the projections of the front-normal velocity, obtained from the four-point timing analysis, onto the $X Y$ GSM plane. Maximum variance directions of the magnetic field are also shown for each probe. The analysis shows that under aforementioned assumptions the flapping fronts propagate in the magnetospheric frame of references dawnward and Earthward roughly perpendicular to the maximum variance direction. The estimated front velocities vary between $13-32 \mathrm{~km} / \mathrm{s}$.

Figure 5 presents ion omni-directional energy-time (ET) spectrograms from both low-energy (ESA, McFadden et al., 2008) and high-energy (SST, Angelopoulos, 2008) instruments, and calculated moments of the ion distribution at three THEMIS probes (P1, P2, and P3). P4 was close to P3 and the plasma characteristics from ESA instruments at both probes were similar, but SST data at P4 were contaminated and could not be used. The plasma sheet was hot during flapping, and high-energy (SST) contribution was included to the moments calculation. The thermal pressure $\left(P_{p}=P_{i}+P_{e}\right)$ is calculated including ion (both ESA and SST) and ESA

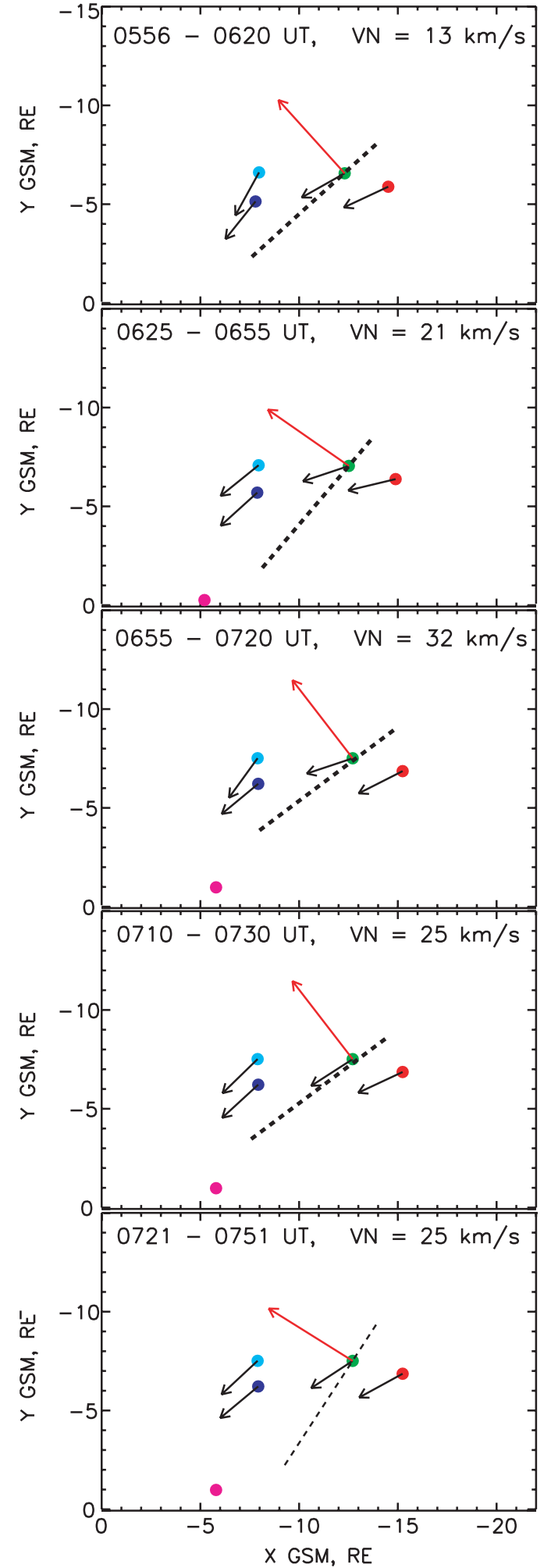

Fig. 4. Orientation and motion of the flapping wave fronts, observed during 06:00-08:00 UT, on 20 December 2007. Black arrows show the magnetic field maximum variance direction, red arrows show the projection of the front normal velocity onto the $X Y$ plane, dashed lines show the front orientation for 5 successive flaps. 

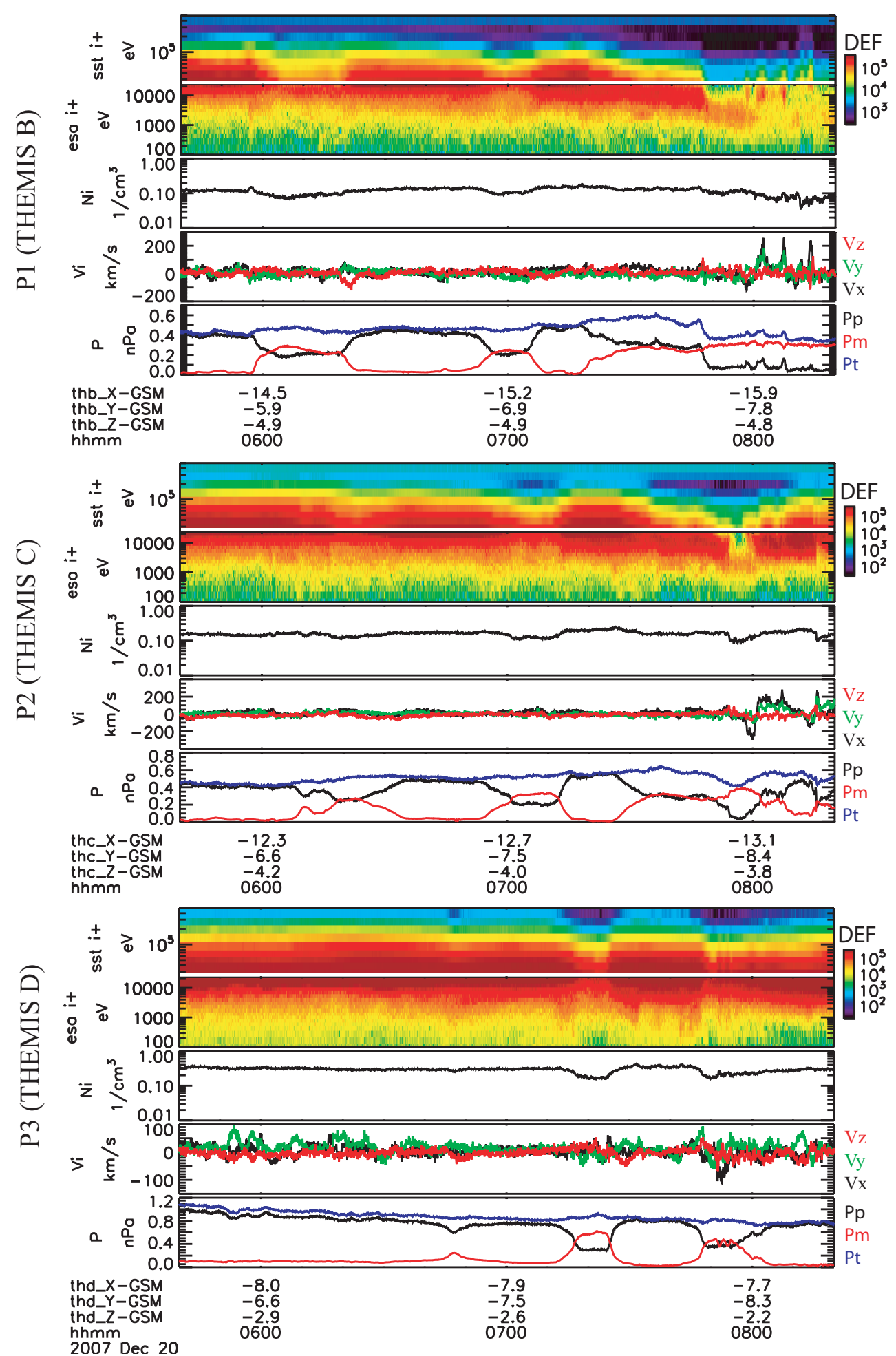

Fig. 5. Ion energy-time omnidirectional spectrograms from SST $(W>25 \mathrm{keV})$ and ESA $(0.01<W<25 \mathrm{keV})$ instruments, the ion number density, GSM components of the ion bulk velocity, magnetic, plasma and total pressures at THEMIS P1, P2, and P3 during 05:40-08:20 UT. The moments are calculated from joint ESA and SST input. 
Table 1. Minimum Variance Analysis of the magnetic field time series during current sheet crossings: the crossing number (\#); the probe used (SC); MVA eigenvalues ( $\lambda_{1}, \lambda_{2}$, and $\lambda_{3}$; the corresponding eigenvectors $\left(\mathbf{R}_{1}, \mathbf{R}_{2}, \mathbf{R}_{3}\right)$ in GSM (excluding for signs); mean values of intermediate $\left(B_{2}\right)$ and normal $\left(B_{3}\right)$ components of the magnetic field during the crossing.

\begin{tabular}{cccccc}
\hline$\#$ & SC & UT & MVA $\lambda_{1,2,3}$ & MVA $_{1}, \mathbf{R}_{2}, \mathbf{R}_{3}$ & $\left|B_{2}\right|,\left|B_{3}\right|$ \\
\hline 1 & P3 & $06: 30: 00-06: 47: 00$ & $32.9,0.54,0.04$ & {$[0.49,0.87,0.07],[0.87,0.48,0.14],[0.09,0.13,0.99]$} & $10.1,11.2$ \\
2 & P1 & $06: 18: 30-06: 24: 00$ & $33.6,0.78,0.02$ & {$[0.97,0.20,0.13],[0.03,0.46,0.89],[0.24,0.87,0.44]$} & $8.7,1.2$ \\
2 & P2 & $06: 28: 00-06: 38: 00$ & $49.4,0.14,0.05$ & {$[0.93,0.39,0.11],[0.09,0.52,0.85],[0.34,0.78,0.52]$} & $9.6,2.5$ \\
2 & P3 & $06: 47: 00-07: 02: 00$ & $27.7,1.22,0.03$ & {$[0.74,0.66,0.10],[0.62,0.74,0.28],[0.26,0.14,0.95]$} & $4.0,13.9$ \\
3 & P2 & $06: 42: 00-07: 02: 00$ & $84.9,0.75,0.17$ & {$[0.89,0.45,0.09],[0.11,0.40,0.91],[0.45,0.80,0.40]$} & $7.7,3.0$ \\
3 & P3 & $07: 09: 00-07: 18: 00$ & $93.1,0.83,0.06$ & {$[0.58,0.80,0.13],[0.27,0.34,0.90],[0.77,0.49,0.41]$} & $11.1,3.0$ \\
4 & P3 & $07: 24: 00-07: 29: 00$ & $90.9,2.51,0.02$ & {$[0.71,0.69,0.14],[0.52,0.38,0.77],[0.47,0.62,0.62]$} & $8.7,4.6$ \\
5 & P1 & $07: 13: 00-07: 21: 00$ & $68.5,1.36,0.45$ & {$[0.88,0.46,0.08],[0.39,0.62,0.68],[0.26,0.63,0.73]$} & $3.7,2.8$ \\
5 & P4 & $07: 38: 00-07: 48: 00$ & $111.3,0.90,0.26$ & {$[0.77,0.63,0.05],[0.47,0.63,0.62],[0.43,0.45,0.78]$} & $7.6,1.7$ \\
\hline
\end{tabular}

electron contributions. Magnetic $\left(P_{m}=B^{2} /\left(2 \mu_{0}\right)\right)$ and total $\left(P_{t}=P_{m}+P_{p}\right)$ pressures are plotted along with the plasma thermal pressure. The observations show that ion velocities during flapping did not exceed $100 \mathrm{~km} / \mathrm{s}$. Variations in the plasma pressure anti-correlated with those of the magnetic pressure, so that the total pressure was fairly conserved during the flapping. The drops of $P_{t}$, observed by $\mathrm{P} 1$ and $\mathrm{P} 2$ at about 07:54 UT, were associated with onset of fast flow and substorm dynamics.

In order to understand the flapping phenomenon, it is important to know how the plasma does move in the flapping wave. For this, in Fig. 6, we plot time series of the maximumvariance magnetic field component and projections of bulk velocity onto the plane, formed by the unit vector in the vertical direction $\left(\mathbf{e}_{Z_{\mathrm{GSM}}}\right)$ and the one in direction, perpendicular to the maximum-variance $\left(\mathbf{e}_{Y^{\prime}}=\mathbf{R}_{\mathbf{1}} \times \mathbf{e}_{Z_{\mathrm{GSM}}}\right)$. The velocity, though small in amplitude, reveals a robust pattern with $V_{z}<0$ when $B_{1}$ is increasing and vice-versa. No constant flow in the $Y^{\prime}$ direction was found. Conversely, $V_{y^{\prime}}$ changes from generally positive (duskward) to somewhat negative (dawnwrad) during the current sheet crossings.

Figure 7 (upper row) presents cross-correlation and linear regression between the main magnetic field change rate $\left(\partial B_{1} / \partial t\right)$ and the Z-component of the ion bulk velocity during the magnetic field variation at P1, P2, and P3. Although the magnetic field change rate was rather low (of $0.1 \mathrm{nT} / \mathrm{s}$, compare with 1-2 nT/s, found by Sergeev et al., 1998), the reasonable anti-correlation was indeed observed. The linear regression slopes may be used to obtain rough estimation of the magnetic field gradient length $(h)$, in the flapping current sheet (Sergeev et al., 1998). Since the magnetic field change rate was found to be small, the gradient length was large (i.e. thick current sheet). The gradient scale was found to be $5000-6000 \mathrm{~km}$ (i.e. $h \approx 1 R_{E}$ ) at the locations of all three probes.

Amplitudes of flapping waves may be estimated by an integration of the apparent displacement of probes with respect to the neutral sheet over time: $S=Z_{0}-\int \delta V_{z} d t$, where $\delta V_{z}$ is the vertical component of the bulk velocity with the longterm trend subtracted, and the initialization constant $Z_{0}$ is set to achieve $S=0$ at the neutral sheet (e.g. Sergeev et al., 2003). Results of the integration at $P 1, P 2$, and $P 3$ are shown in Fig. 7 (bottom panel). Curves at P1 and P2 are of a similar shape, showing the amplitudes of 2 and $4 R_{E}$ for the first and the second fronts, respectively, and smaller amplitudes for 3rd, 4th, and 5th fronts. Since the amplitude of the magnetic field variations remained the same (it even increased slightly at fronts 4 and 5, see Fig. 3), the current sheet at $X=12-15 R_{E}$ became thinner. The $\mathrm{P} 3$ trace shows, however, a smaller amplitude for the 1st front and somewhat larger amplitudes for 3rd and 4th ones.

\section{Summary and discussion}

In this paper we report on the observation of large amplitude magnetic field variations with a quasi-period of 20 40 min observed by four THEMIS probes situated in the plasma sheet at $-8>X>16 R_{E}$ within $-6.5>Y>-7.5 R_{E}$. Although the period of the magnetic field oscillations was larger than that discussed in the context of Cluster observations of rapid current sheet flapping (see Sharma et al., 2008, for review), the basic properties of the waves, observed by THEMIS are similar to flapping waves. Namely, the wave fronts (assuming the planarity) were found to be propagating flankward at a velocity of $20 \mathrm{~km} / \mathrm{s}$ with respect to the spacecraft; the normal to the current sheet was tilted in the $Y^{\prime} Z$-plane; the anti-correlation between the main magnetic field change rate $\left(\partial B_{1} / \partial t\right)$ and the vertical component of the bulk velocity $v_{z}$ was observed; the amplitude of the wave was estimated to be of $1-3 R_{E}$. Thus the observed magnetic field variations were due to large-scale corrugations or tail-elongated ripples on the current sheet surface, crossed by the probes. Such structures of the current sheet were predicted by phenomenological model of "slippage"-mode flapping (Petrukovich et al., 2006) as well as by analytical models of the flapping waves (Golovchanskaya and Maltsev, 

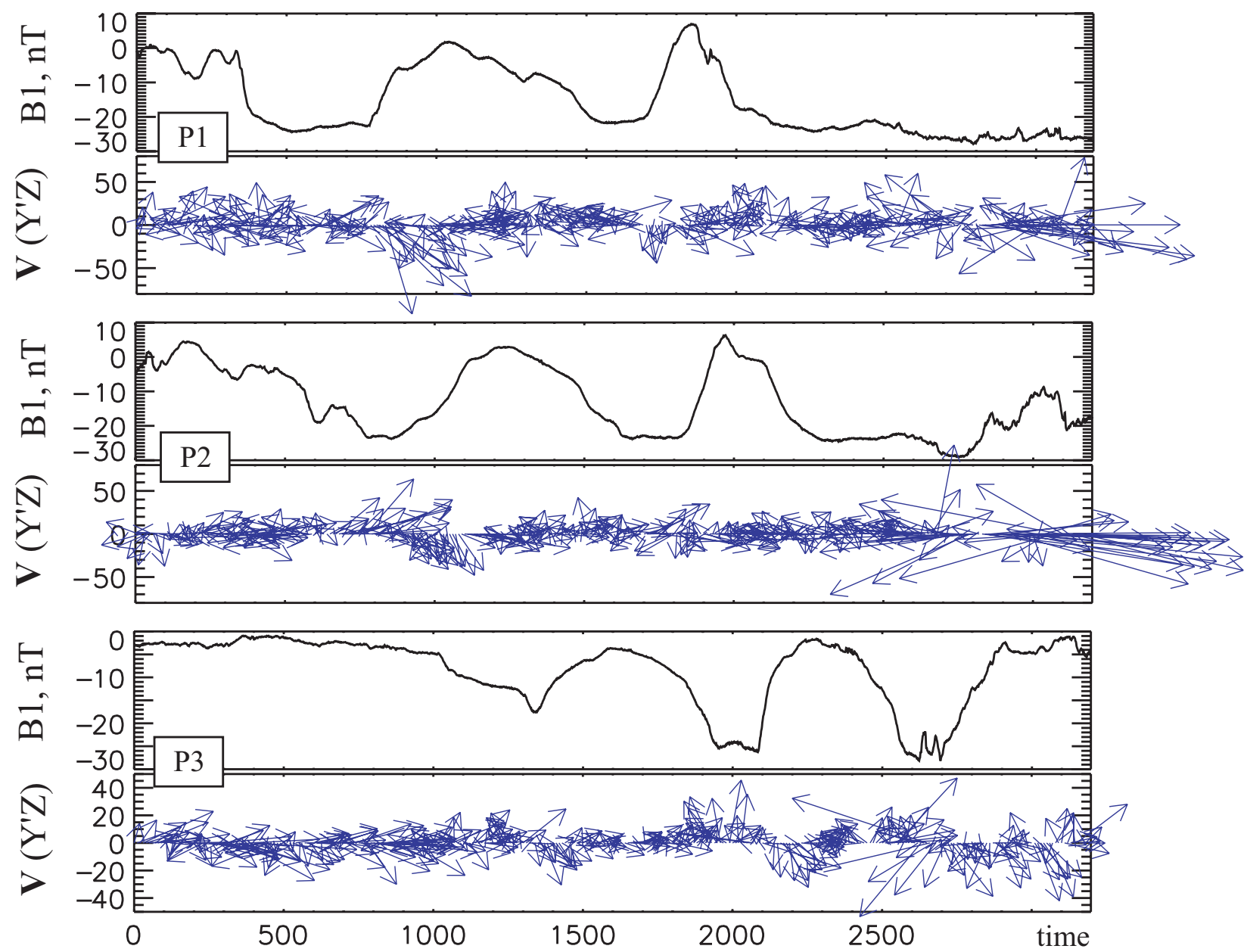

Fig. 6. Maximum variance component of the magnetic field $\left(B_{1}\right)$ and projections of plasma bulk velocity onto the plane perpendicular to $B_{1}$ $(Y, Z)$ versus time (arbitrary units).

2004; Erkaev et al., 2008). No high-speed plasma flows were observed at the THEMIS location during the magnetic field variations (flapping). The total pressure (a sum of the magnetic and plasma thermal pressures) was found to be nearly constant during the magnetic field oscillations, which indicates the non-compressional nature of the flapping waves. The plasma motion in the observed flapping waves was found to be involved into vertical up-down and reversing horizontal motions. Such an intermittent behavior of the bulk velocity during flapping resembles the vortex-like plasma motion, predicted by the double-gradient model (Erkaev et al., 2008). Since no quasi-steady dawnward plasma flow was observed, flapping, in the studied case, was due to the "real" wave propagation through ambient plasma, but not due to "frozen-in" magnetic field folds, transported by the background plasma flow (Sergeev et al., 2006a).

Was the observed flapping induced by solar wind/IMF dynamics? In the reported case, the magnetic field variations observed in the plasma sheet were preceded by a significant variation of IMF $B_{y}$ between 05:00-06:00 UT, observed by all three spacecraft providing IMF measurements (Fig. 1). A significant IMF compression was also detected by Geotail (but was not observed either by ACE or by WIND) between 05:45-06:00 UT. WIND also showed solar wind $V_{z}$ variations with amplitudes of $20 \mathrm{~km} / \mathrm{s}$ (which is comparable to the $V_{z}$-variation amplitude used by Sergeev et al. (2008) to simulate the magnetotail response). Whether these variations in solar wind and IMF trigger magnetotail current sheet flapping is to be addressed to modeling.

Was flapping related to magnetic activity? The flapping oscillations, analyzed in the presented event studies, were observed by THEMIS spacecraft between two significant peaks of the pseudo-AE, calculated using THEMIS groundbased magnetometers (Fig. 1), i.e. during a local minimum of AE. However, the X-component of the magnetic field, measured by stations situated near the THEMIS probes foot points, showed a continuous negative trend modulated by 20-40 min long bay-like variations between 06:00-07:40 UT (Fig. 2). These signatures may be interpreted as a slow buildup of westward electrojet, preceding a substorm-associated 

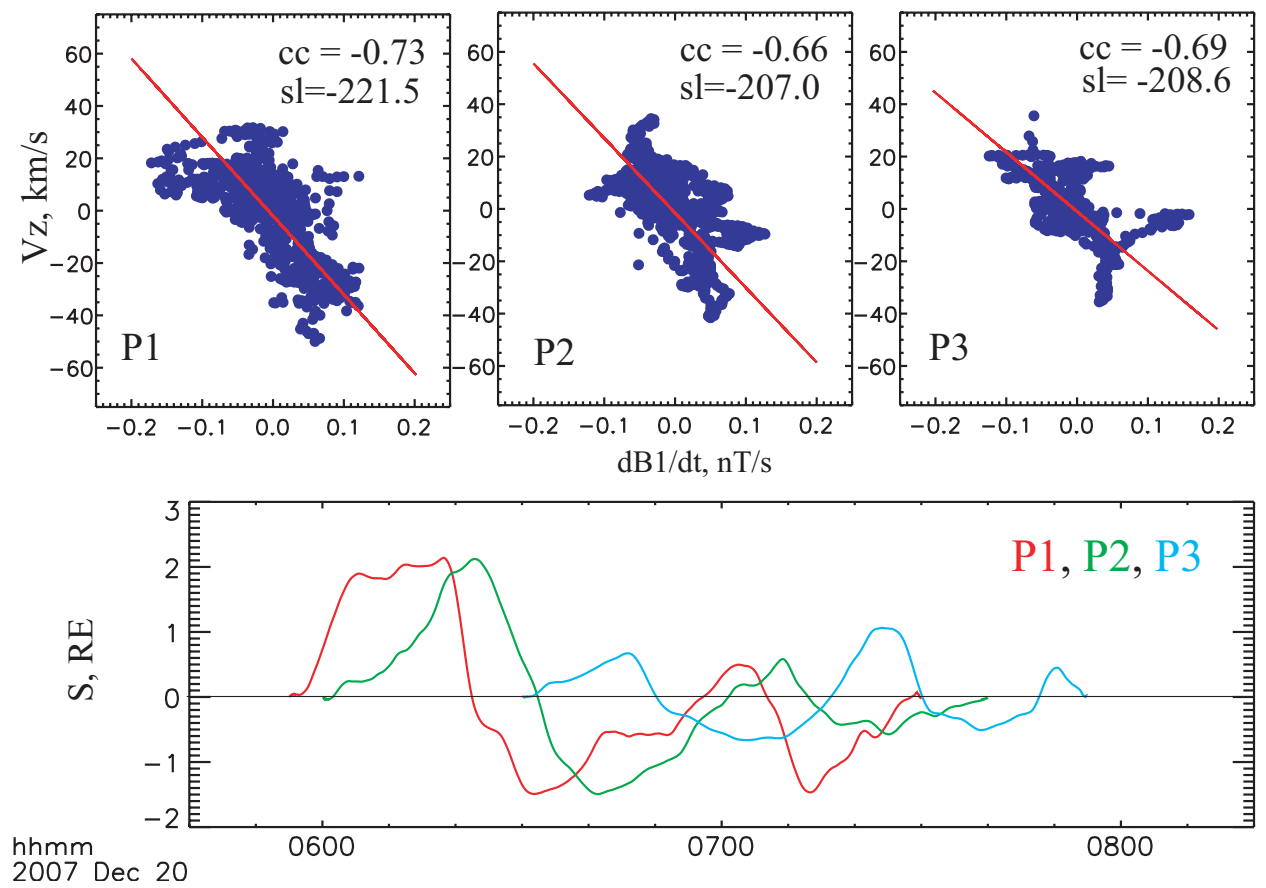

Fig. 7. Comparison of the main magnetic field variation rate $\left(\partial B_{1} / \partial t\right)$ and the vertical bulk velocity $\left(V_{z}\right)$ at $\mathrm{P} 1, \mathrm{P} 2$, and $\mathrm{P} 3$ during flapping intervals (upper row). Integrated $V_{z}$ versus UT (bottom panel).

$B_{x}$ bay between 07:40-08:30 UT. Thus, the observations suggest that the flapping oscillations appeared during the substorm growth phase. This conclusion is also supported by in-situ measurements: both $\mathrm{P} 3$ and $\mathrm{P} 4$, situated near geosynchronous orbit, show a gradual decrease of $B_{z}$, i.e. a tail field stretching (likely caused by southward IMF turning at about 04:10 UT), until about 08:00 UT (Fig. 1). Some thinning of the cross-tail current sheet was also found (see Fig. 7 and corresponding discussion).

\section{Concluding remarks}

The presented event study reveals certain features of flapping as a large-scale process in the magnetospheric plasma sheet.

1. The tail-aligned scale of flapping corrugation is larger than separation of THEMIS spacecraft $\left(7 R_{E}\right)$.

2. An individual fold may survive as long as 20 min without large changes in its shape. Its half-width was estimated to be $2-3 R_{E}$, i.e. roughly equal to the estimated vertical amplitude. Thus the flapping corrugations are steep tail-elongated ridges with the cross-scale in an order of magnitude smaller than the lengthwise one.

3. Simultaneous observations in space and on the ground show that flapping was observed during the growth phase of magnetospheric activity.
Thus, the event study, presented in this paper, shows that the THEMIS mission may bring the valuable information required to attack fundamental problems on flapping current sheet structure, dynamics and the relations between flapping and magnetospheric activity.

Acknowledgements. This work was supported by NASA grant NAS5-0299 and German Ministry for Economy and Technology and the German Center for Aviation and Space (DLR), contract 50QP0402. ACE and WIND data are provided by CDAWeb; SC ground tracks are provided by SSCWeb. We thank P. Cruce, B. Kerr, and A. Prentice for the help with software and editions.

Topical Editor R. Nakamura thanks M. Kuznetsova and another anonymous referee for their help in evaluating this paper.

\section{References}

Angelopoulos, V.: The THEMIS Mission, Space Sci. Rev., 141, 534, doi: 10.1007/s11214-008-9336-1, 2008.

Auster, H. U., Glassmeier, K. H., Magnes, W., et al.: The THEMIS Fluxgate Magnetometer, Space Sci. Rev., 141, 235-264, doi: 55610.1007/s11214-008-9365-9, 2008.

Erkaev, N. V., Semenov, V. S., and Biernat, H. K.: Magnetic double gradient mechanism for flapping oscillations of a current sheet, Geophys. Res. Lett, 35, L02111, doi:10.1029/2007GL032277, 2008.

Gabrielse, C., Angelopoulos, V., Runov, A., Kepko, L., Glassmeier, K.-H., Auster, H. U., McFadden, J., Carlson, C. W., and Larson, D.: Propagation characteristics of plasma sheet oscillations during a small storm, Geophys. Res. Lett., 35, L17S13, doi: 10.1029/2008GL033664, 2008. 
Golovchanskaya, I. V. and Maltsev, Y. P.: On the identification of plasma sheet flapping waves observed by Cluster, Geophys. Res. Lett., 32, L02102, doi:10.1029/2004GL021552, 2004.

Harvey, C. C.: Spatial gradients and volumetric tensor, in: Analysis Methods for Multi-Spacecraft Data, edited by: Paschmann, G. and Daly, P., pp. 307-322, ESA, Noordwijk, 1998.

Lui, A. T. Y., Meng, C.-I., and Akasofu, S.-I.: Wavy nature of the magnetotail neutral sheet, Geophys. Res. Lett., 5, 279-282, 1978.

Mann, I. R., Milling, D. K., Rae, I. J., et al.: The upgraded CARISMA magnetometer array in the THEMIS era, Space Sci. Rev.., 141, 413-451, doi:10.1007/s11214-008-9380-x, 2008.

McComas, D. J., Russel, C. T., Elphic, R. C., and Bame, S. J.: The near-Earth cross-tail current sheet: Detailed ISEE 1 and 2 case studies, J. Geophys. Res., 91, 4287-4301, 1986.

McFadden, J. P., Carlson, C. W., Larson, D., Angelopolos, V., Ludlam, M., Abiad, R., and Elliot, B.: The THEMIS ESA Plasma Instrument and In-flight Calibration, Space Sci. Rev., 141, 277 302, doi:10.1007/s11214-008-9440-2, 2008.

Mende, S. B., Harris, S. E., Frey, H. U., Angelopoulos, V., Russell, C. T., Donovan, E., Jackel, B., Greffen, M., and Peticolas, L. M.: The THEMIS Array of Ground-based Observatories for the Study of Auroral Substorms, Space Sci. Rev., 141, 357-387, doi:10.1007/s11214-008-9380, 2008.

Nakagawa, T. and Nishida, A.: Southward magnetic field in the neutral sheet produced by wavy motions propagating in the dawndusk direction, Geophys. Res. Lett., 16, 1265-1268, 1989.

Petrukovich, A. A., Baumjohann, W., Nakamura, R., Balogh, A., Mukai, T., Glassmeier, K.-H., Rème, H., and Klecker, B.: Plasma sheet structure during strongly northward IMF, J. Geophys. Res., 108, 1258, doi:10.1029/2002JA009738, 2003.

Petrukovich, A. A., Zhang, T. 1., Baumjohann, W., Nakamura, R., Runov, A., Balogh, A., and Carr, C.: Oscillatory magnetic flux tube slippage in the plasma sheet, Ann. Geophys., 24, 16951704, 2006, http://www.ann-geophys.net/24/1695/2006/.

Press, W. H., Teukolsky, S. A., Vetterling, W. T., and Flannery, B. P.: Numerical recipes in C. The art of scientific computing, Cambridge: University Press, 2nd ed., 1992.

Runov, A., Sergeev, V. A., Baumjohann, W., Nakamura, R., Apatenkov, S., Asano, Y., Volwerk, M., Vörös, Z., Zhang, T. L., Petrukovich, A., Balogh, A., Sauvaud, J.-A., Klecker, B., and Rme, H.: Electric current and magnetic field geometry in flapping magnetotail current sheets, Ann. Geophys., 23, 1391-1403, 2005, http://www.ann-geophys.net/23/1391/2005/.

Runov, A., Sergeev, V. A., Nakamura, R., Baumjohann, W., Apatenkov, S., Asano, Y., Takada, T., Volwerk, M., Vörös, Z., Zhang, T. L., Sauvaud, J.-A., Rème, H., and Balogh, A.: Local structure of the magnetotail current sheet: 2001 Cluster observations, Ann. Geophys., 24, 247-262, 2006, http://www.ann-geophys.net/24/247/2006/.
Sergeev, V., Angelopulous, V., Carlson, C., and Sutcliffe, P.: Current sheet measurements within a flapping plasma sheet, J. Geophys. Res., 103, 9177-9188, 1998.

Sergeev, V., Runov, A., Baumjohann, W., et al.: Current sheet flapping motion and structure observed by Cluster, Geophys. Res. Lett., 30, 1327, doi:10.1029/2002GL016500, 2003.

Sergeev, V., Runov, A., Baumjohann, W., Nakamura, R., Zhang, T. L., Balogh, A., Louarn, P., Sauvaud, J.-A., and Rème, H.: Orientation and propagation of current sheet oscillations, Geophys. Res. Lett., 31, L05807, doi:10.1029/2003GL019346, 2004.

Sergeev, V., Runov, A., Baumjohann, W., Nakamura, R., Zhang, T. L., Apatenkov, S., Balogh, A., Rème, H., and Sauvaud, J.A.: Cluster results on the magnetotail current sheet structure and dynamics, in: Proceedings Cluster and Double Star Symposium 5th Anniversary of Cluster in Space, p. 9.2, ESA SP-598, 2006 a.

Sergeev, V. A., Sormakov, D. A., Apatenkov, S. V., Baumjohann, W., Nakamura, R., Runov, A. V., Mukai, T., and Nagai, T.: Survey of large-amplitude flapping motions in the midtail current sheet, Ann. Geophys., 24, 2015-2024, 2006b.

Sergeev, V. A., Tsyganenko, N. A., and Angelopoulos, V.: Dynamical response of the magnetotail to changes of the solar wind direction: an MHD modeling perspective, Ann. Geophys., 26, 2395-2402, 2008, http://www.ann-geophys.net/26/2395/2008/.

Sharma, A. S., Nakamura, R., Runov, A., Grigorenko, E. E., Hasegawa, H., Hoshino, M., Louarn, P., Owen, C. J., Petrukovich, A., Sauvaud, J.-A., Semenov, V. S., Sergeev, V. A., Slavin, J. A., Sonnerup, B. U. Ö., Zelenyi, L. M., Fruit, G., Haaland, S., Malova, H., and Snekvik, K.: Transient and localized processes in the magnetotail: a review, Ann. Geophys., 26, 9551006, 2008, http://www.ann-geophys.net/26/955/2008/.

Sonnerup, B. U. Ö. and Scheible, M.: Minimum and maximum variance analysis, in: Analysis Methods for Multi-Spacecraft Data, edited by: Paschmann, G. and Daly, P., pp. 185-220, ESA, Noordwijk, 1998.

Speiser, T. W. and Ness, N. F.: The neutral sheet in the geomagnetic tail: Its motion, equivalent currents, and field line reconnection through it, J. Geophys. Res., 72, 131-141, 1967.

Toichi, T. and Miyazaki, T.: Flapping motions of the tail plasma sheet induced by the interplanetary magnetic field variations, Planet. Space Sci., 24, 147-159, 1976.

Zhang, T. L., Baumjohann, W., Nakamura, R., Balogh, A., and Glassmeier, K.-H.: A wavy twisted neutral sheet observed by Cluster, Geophys. Res. Lett., 29, 1899, doi:10.1029/ 2002GL015544, 2002.

Zhang, T. L., Nakamura, R., Volwerk, M., Runov, A., Baumjohann, W., Eichelberger, H. U., Carr, C., Balogh, A., Sergeev, V., Shi, J. K., and Fornacon, K.-H.: Double Star/Cluster observation of neutral sheet oscillations on 5 August 2004, Ann. Geophys., 23, 2909-2914, 2005, http://www.ann-geophys.net/23/2909/2005/. 